\title{
Accurate measurement of the time delay in the response of the LIGO gravitational wave detectors
}

\author{
Yoichi Aso ${ }^{1,2}$, Evan Goetz ${ }^{3}$, Peter Kalmus ${ }^{1}$, Luca Matone ${ }^{1}$, \\ Szabolcs Márka ${ }^{1}$, Joshua Myers ${ }^{5}$, Brian O'Reilly ${ }^{4}$, Rick Savage, \\ Paul Schwinberg ${ }^{5}$, Xavier Siemens ${ }^{6}$, Daniel Sigg $^{5}$ and Nicolas Smith \\ ${ }^{1}$ Department of Physics, Columbia University, New York, NY 10027, USA \\ 2 LIGO Laboratory, California Institute of Technology, Pasadena, CA 91125, USA \\ ${ }^{3}$ University of Michigan, Ann Arbor, MI 48109, USA \\ ${ }^{4}$ LIGO Livingston Observatory, Livingston, LA 70754, USA \\ 5 LIGO Hanford Observatory, Richland, WA 99352, USA \\ ${ }^{6}$ Department of Physics, University of Wisconsin at Milwaukee, Milwaukee, WI 53211, USA \\ ${ }^{7}$ LIGO Laboratory, Massachusetts Institute of Technology, Cambridge, MA 02139, USA \\ E-mail: aso@caltech.edu
}

Received 8 September 2008, in final form 28 December 2008

Published 17 February 2009

Online at stacks.iop.org/CQG/26/055010

\begin{abstract}
We present a method to precisely calibrate the time delay in a long baseline gravitational-wave interferometer. An accurate time stamp is crucial for data analysis of gravitational wave detectors, especially when performing coincidence and correlation analyses between multiple detectors. Our method uses an intensity-modulated radiation pressure force to actuate on the mirrors. The time delay is measured by comparing the phase of the signal at the actuation point with the phase of the recorded signal within the calibrated data stream used for gravitational wave searches. Because the signal-injection path is independent of the interferometer's control system, which is used for the standard calibration, this method can be an independent verification of the timing error in the system. A measurement performed with the $4 \mathrm{~km}$ interferometer at the LIGO Hanford Observatory shows a $1 \mu$ s relative accuracy when averaging over $50 \mathrm{~min}$. Our understanding of the systematic time delay in the detector response has reached the level of $10 \mu \mathrm{s}$.
\end{abstract}

PACS numbers: 95.55.Ym, 04.80.-y

\section{Introduction}

The Laser Interferometer Gravitational-wave Observatory (LIGO) is a network of gravitational wave [1] detectors consisting of three long baseline interferometers [2] at two sites near Hanford, Washington and Livingston, Louisiana [3]. On 1 October 2007, LIGO finished its 
fifth science run (S5), which collected one year of triple coincidence data at design sensitivity [4]. LIGO is looking for transient gravitational wave sources such as the coalescence of compact binary stars and the explosion of supernovae. LIGO also looks for continuous gravitational wave emissions from asymmetric pulsars and for a stochastic background in gravitational waves from the early universe. In order to find weak gravitational wave signals hidden in a noisy background good timing accuracy of the measured detector signals is required. For example, in the search for transient gravitational wave signals one compares the outputs from multiple detectors and looks for a consistent signature [5-7]. If the time of the events is not very well known, one can falsely reject real signals. In other cases, we look for coincident events with other astrophysical detectors such as gamma-ray and optical telescopes [8-13]. This requires an absolute time stamp. When searching for continuous gravitational wave emissions from pulsars, one coherently integrates the signal over a long time period to improve the signal-to-noise ratio $[14,15]$. An inaccurate time stamp within the detector network can de-phase the intrinsically coherent signal and thus degrade the signalto-noise ratio. When searching for a stochastic gravitational wave background, one computes the correlation between multiple detectors [16], again, requiring accurate timing to avoid de-phasing.

This paper quantifies the accuracy and provides a detailed description of a method that can be used to precisely measure the time delay between a simulated gravitational wave force on an interferometer end test mass and the calibrated data stream used for gravitational wave searches. This method uses a sinusoidally modulated laser beam illuminated on a test mass mirror to apply a varying force by changing the radiation pressure. This signal-injection system is called the photon calibrator [17]. The induced change in the differential arm length mimics the effect of a gravitational wave. The resultant signal at the anti-symmetric port of the interferometer is digitized, recorded and calibrated providing input for data analysis. By determining the relative phase between the calibrated data stream and the injected signal, one can measure the time delay in the interferometer. By taking into account the absolute time stamps and by using the calibrated strain or displacement signal, one can also independently verify the end-to-end accuracy of the timing and phase calibration.

Using only a single-frequency sinusoid would limit our ability to measure the time delay to less than the period of the sinusoid. To mitigate this limitation one can choose a lower frequency. However, this will reduce the accuracy in two ways: the sensitivity of the interferometer gets worse at very low frequencies and a longer period leads to larger timing errors. We chose to extend the range of the time-delay measurement by injecting two closely spaced sinusoids. Careful comparison of phase delays at the two frequencies enables us to measure the time delay for a much wider range, up to $\pm 1 /(2 \Delta f)$, where $\Delta f$ denotes the difference between the two frequencies.

There are two types of uncertainties we consider: (a) the accuracy of the synchronization of the analog-to-digital converters (ADCs), which were used to record the signals, relative to universal coordinate time (UTC) as measured by a GPS clock, and (b) the intrinsic time delay within the instrument, which has to be accounted for by the phase calibration of the detector.

\section{Measurement}

The schematic diagram of the photon calibrator is shown in figure 1. It consists of a $500 \mathrm{~mW}$ $\mathrm{Nd}$ :YLF laser at a wavelength of $1047 \mathrm{~nm}$. An acousto-optic modulator (AOM) is used to modulate the laser intensity. The maximum modulation depth is about $50 \%$. A small part of the beam is split off towards a photodetector which is used to monitor the injected signal. 


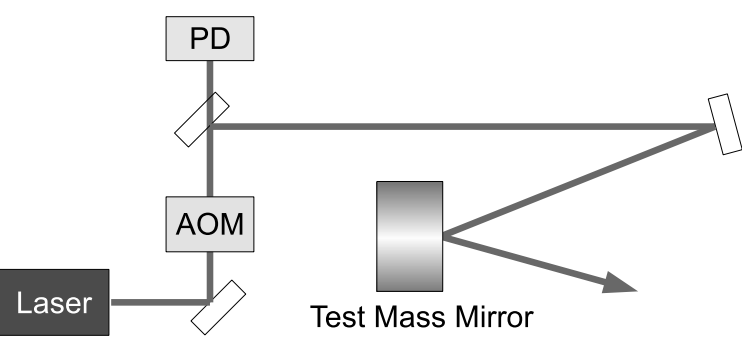

Figure 1. Conceptual diagram of the photon calibrator. AOM: acousto-optic modulator, PD: photo detector.

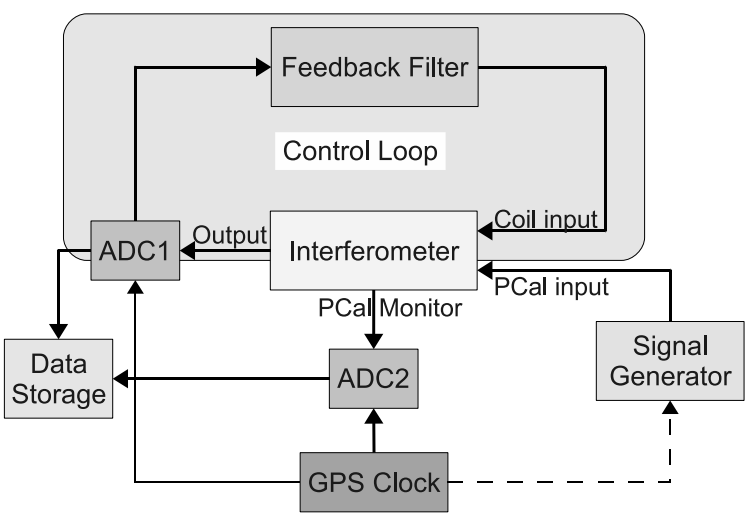

Figure 2. Block diagram of the measurement. ADC: analog-to-digital converter.

Most of the beam is sent into the vacuum system and is bounced off the front surface of an end test mass. Radiation pressure and, hence, the force on the mirror is proportional to the incident laser power. By varying the amplitude of the signal driving the AOM, one can inject any desired waveform.

One can assume that the actuation on the end mirrors is equivalent to the effect of a gravitational wave as far as the wavelength of the gravitational wave is large enough compared to the size of the detector [18]. This condition is well satisfied for most of the searches being conducted with the LIGO data, which use frequency bands below a few $\mathrm{kHz}$. When searching for high-frequency gravitational waves, one has to take into account the corrections arising from the difference of the interferometer's response to the end-mirror motion and gravitational waves [19].

The whole measurement setup is shown as a block diagram in figure 2. In this simplified picture, a LIGO interferometer is treated as a 2-input 2-output device. The first input to the interferometer is the coil magnet actuators on the mirrors of the interferometer. These actuators are used to keep the mirrors in the proper operating positions by the length control loop (marked by a grey box in figure 2) [20]. Another input is the modulation drive of the photon calibrator. The anti-symmetric output from the interferometer which will contain the gravitational wave signals is fed into ADC1 and recorded. The output signal from the interferometer is affected by the the control loop. Therefore, the gravitational waveform has to be reconstructed from the displacement calibration. This calibrated output of the interferometer is often called $h(t)$ 
in the gravitational wave community, and we will use this convention in this paper. The output from the monitor photodetector of the photon calibrator is recorded by ADC2. The ADCs are synchronized to a GPS clock with high accuracy through LIGO's timing distribution system. The accuracy and offset of the ADC time stamp was measured independently, and correction factors were included for this analysis.

The signal generator produced a superposition of two sinusoids (two-tone or DuoTone signal) at $110 \mathrm{~Hz}$ and $111 \mathrm{~Hz}$. This two-tone signal was then injected into the interferometer using the photon calibrator. The frequencies were chosen to be in the most sensitive band of the interferometer and to be away from disturbing noise lines, such as power line harmonics. The experiment was performed on the $4 \mathrm{~km}$ interferometer at the LIGO Hanford Observatory. For about $10 \mathrm{~h}$ the signal was applied to the end test mass of the in-line arm. This measurement was done just after S5, when the LIGO interferometer was still in the same configuration as during the science run.

\section{Time-delay analysis}

The analysis of the recorded two-tone signals measures the time delay between the interferometer's main output, $h(t)$, and the photon calibrator monitor photodetector. We denote the photon-calibrator monitor signal $p(t)$. We used $p(t)$ as the reference for the timedelay measurement. The time delay between $p(t)$ and the actual time of the radiation pressure force applied to the mirror is supposed to be small. We will take into account a small delay in the photo detector and the timing correction for ADC2 when interpreting the results in section 6.

The time delay was determined by measuring the relative phase between $h(t)$ and $p(t)$. For a pure sinusoid at fixed frequency $\omega$ the phase delay, $\delta \phi$, and the time delay, $\delta t$, are related by $\delta \phi=\omega \delta t$. For a single sinusoid, a constant time delay corresponds to a phase which grows proportionally with frequency. Linear filters, such as those used in the instrument, will in general not look like a constant delay. Therefore, it is convenient to decompose the phase delay into two components: (a) a phase delay due to a time delay which has linear frequency dependency and (b) a residual phase delay which is not proportional to frequency.

\subsection{Amplitude normalization and sine wave extraction}

To measure the phase of one frequency component of the two-tone signal, we put $h(t)$ or $p(t)$ through a zero-phase [21] band-pass filter of eighth order (the FWHM of the pass band is about $0.25 \mathrm{~Hz}$ ). Zero phase filtering was realized by applying a fourth-order infiniteimpulse-response (IIR) filter back and forth to cancel the phase change of each filter. After the filtering, we normalized the amplitude of the sinusoid to average out the low-frequency noise contribution. For this purpose, we multiplied the signal with two quadratures, $\sin (\omega t)$ and $\cos (\omega t)$. To get the amplitude variation of the input sinusoid, the resultant time series were quadratically added and filtered through a fourth-order low-pass filter with a corner frequency of $3 \mathrm{~Hz}$. The original signal is then normalized by this amplitude.

\subsection{Reconstruction of time delay and phase difference}

Our injected signal is a superposition of two sinusoids with unknown phase offset $\Psi$,

$$
S_{\text {inj }}(t)=\sin \left(\omega_{1} t\right)+\sin \left(\omega_{2} t+\Psi\right) .
$$

We know the frequencies, $\omega_{1}$ and $\omega_{2}$, precisely. This signal is split into two paths leading to $h(t)$ and $p(t)$. Since these paths have different delays, $h(t)$ and $p(t)$ can be written as 


$$
\begin{aligned}
& h(t)=\sin \left[\omega_{1}\left(t+\Delta t_{\mathrm{h}}\right)\right]+\sin \left[\omega_{2}\left(t+\Delta t_{\mathrm{h}}\right)+\phi_{\mathrm{h}}+\Psi\right], \\
& p(t)=\sin \left[\omega_{1}\left(t+\Delta t_{\mathrm{p}}\right)\right]+\sin \left[\omega_{2}\left(t+\Delta t_{\mathrm{p}}\right)+\phi_{\mathrm{p}}+\Psi\right] .
\end{aligned}
$$

Here, $\Delta t_{\mathrm{h}}$ and $\Delta t_{\mathrm{p}}$ are the frequency-independent time delay of the two paths and $\phi_{\mathrm{h}}$ and $\phi_{\mathrm{p}}$ are the residual phase delays which are not proportional to frequency. Since we are only interested in phases, we ignored the amplitudes. Our objective is to measure the time delay $\delta t \equiv \Delta t_{\mathrm{h}}-\Delta t_{\mathrm{p}}$ and the phase difference $\delta \phi \equiv \phi_{\mathrm{h}}-\phi_{\mathrm{p}}$ independently.

After extracting the $\omega_{1}$ component of the signals by sending $h(t)$ and $p(t)$ through the narrow band-pass filter described in the previous section, we obtain

$$
\begin{aligned}
& h_{\omega_{1}}(t)=\sin \left[\omega_{1}\left(t+\Delta t_{\mathrm{h}}\right)\right], \\
& p_{\omega_{1}}(t)=\sin \left[\omega_{1}\left(t+\Delta t_{\mathrm{p}}\right)\right] .
\end{aligned}
$$

The phase of each signal is measured by the three parameter sine wave fitting algorithm described by IEEE standard 1057 [22, 23]. The relative phase $\theta_{1}$ between $h_{\omega_{1}}(t)$ and $p_{\omega_{1}}(t)$ is obtained as

$$
\theta_{1}=\omega_{1} \delta t \bmod 2 \pi .
$$

Because of the periodicity of the signals, $\theta_{1}$ is obtained as the common residue of the true relative phase $\omega_{1} \delta t$ modulo $2 \pi$. Here our convention of the modulo operation is $a \bmod b \equiv \operatorname{sgn}(b)|a-\lfloor a / b\rfloor \cdot b|$, so that the obtained phase is always between 0 and $2 \pi(\lfloor x\rfloor$ means rounding operation of $x$ towards $-\infty)$. Similarly, we can obtain the relative phase between $h(t)$ and $p(t)$ at frequency $\omega_{2}$ as

$$
\theta_{2}=\left(\omega_{2} \delta t+\delta \phi\right) \bmod 2 \pi \text {. }
$$

By taking the difference between the above two quantities, we obtain

$$
\delta \theta=\left(\theta_{2}-\theta_{1}\right) \bmod 2 \pi=(\delta \omega \delta t+\delta \phi) \bmod 2 \pi,
$$

where $\delta \omega \equiv \omega_{2}-\omega_{1}$. Now, if we assume that $|\delta \omega \delta t+\delta \phi|$ is smaller than $\pi$, we get

$$
\delta \theta= \begin{cases}\delta \omega \delta t+\delta \phi & \text { for } \quad(\delta \omega \delta t+\delta \phi) \geqslant 0 \\ \delta \omega \delta t+\delta \phi+2 \pi & \text { for } \quad(\delta \omega \delta t+\delta \phi)<0 .\end{cases}
$$

$h(t)$ and $p(t)$ should only be different by a factor of $-2 \cos \alpha / c M \omega^{2} P$, where $P$ is the incident laser power amplitude, $\alpha$ is the angle of incident and $M$ is the mass of the mirror. As a consequence, $\delta \phi-\pi$ should be equal to zero or at least very small. We have eliminated the $\pi$-phase shift from the following calculations by simply redefining $p(t) \rightarrow-p(t)$.

From (6) we see that $\delta t=\left(\theta_{1}+2 \pi n\right) / \omega_{1}$, where $n$ is an integer. Since we do not know this integer beforehand, we try $\delta t_{k}=\left(\theta_{1}+2 \pi k\right) / \omega_{1}\left(k\right.$ is the trial integer) and subtract $\delta \omega \delta t_{k}$ from $\delta \theta$. When $(\delta \omega \delta t+\delta \phi) \geqslant 0$, we get

$$
\delta \phi_{k} \equiv \delta \theta-\delta \omega \delta t_{k}=\delta \omega\left(\delta t-\delta t_{k}\right)+\delta \phi=\frac{\delta \omega}{\omega_{1}} 2 \pi(n-k)+\delta \phi .
$$

Here $\delta \phi_{k}$ is at the minimum, $\delta \phi_{k}=\delta \phi$, at $k=n$ assuming $\delta \phi<\pi \delta \omega / \omega_{1}$. Therefore, by scanning $k$ for the minimum $\delta \phi_{k}$ we can find the true value of $n$ and consequently $\delta t$ and $\delta \phi$. When $(\delta \omega \delta t+\delta \phi)<0$, we get

$$
\delta \phi_{k}=\frac{\delta \omega}{\omega_{1}} 2 \pi\left(n-k+\frac{\omega_{1}}{\delta \omega}\right)+\delta \phi .
$$

Here $\delta \phi_{k}$ is at its minimum at $k=n^{\prime} \equiv n+\omega_{1} / \delta \omega$. For $k=n^{\prime}$, we get $\delta t_{n^{\prime}}=\delta t+2 \pi / \delta \omega$. Here we assume that $|\delta t|$ is much smaller than $\pi / \delta \omega$, so that $\delta t_{n^{\prime}}$ is always larger than $\pi / \delta \omega$. 


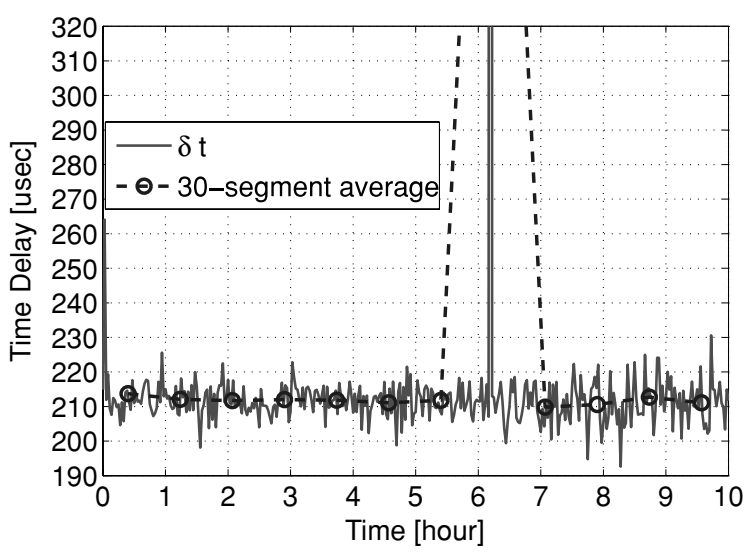

Figure 3. The measured time delays between the photon calibrator photodetector and the calibrated output of the interferometer.

Then, if the $\delta t_{k}$ found by the minimization of $\delta \phi_{k}$ is greater than $\pi / \delta \omega$, we can conclude that $(\delta \omega \delta t+\delta \phi)<0$ and the true $\delta t$ is $\delta t_{k}-2 \pi / \delta \omega$.

In the above section we made three assumptions. The assumption $|\delta t| \ll \pi / \delta \omega$ means that $|\delta t|$ is shorter than the half-period of $\delta \omega$. For our experiment, $\pi / \delta \omega$ is $0.5 \mathrm{~s}$. Since $\delta t$ is expected to be much smaller than this value (most likely less than a millisecond), we can safely assume this. The assumption on $\delta \phi$ that $\delta \phi<\pi \delta \omega / \omega_{1}$ is justified by the fact that the frequencies of the two sinusoids are very close and that there should be no phase shift in the first place. In fact, for our experiment this requirement leads to $\delta \phi<1.64^{\circ}$ between $110 \mathrm{~Hz}$ and $111 \mathrm{~Hz}$. This rate of phase change is larger than the steepest part of the phase change by a first-order low-pass filter with a cut-off frequency of $100 \mathrm{~Hz}$. The calibrated response of the interferometer should be much flatter than a first-order low-pass filter. Finally, the remaining assumption, $|\delta \omega \delta t+\delta \phi|<\pi$, is automatically satisfied by the other two assumptions.

\section{Results}

Figures 3 and 4 show the measured $\delta t=\Delta t_{\mathrm{h}}-\Delta t_{\mathrm{p}}$ and $\delta \phi=\Delta \phi_{\mathrm{h}}-\Delta \phi_{\mathrm{p}}$. The $10 \mathrm{~h}$ long data set was divided into $100 \mathrm{~s}$ long segments, and $\delta t$ and $\delta \phi$ were determined for each segment. The curves labelled '30-segment average' are averages over 30 consecutive segments. The convention here is that the positive value of $\delta t$ means $h(t)$ is advanced from $p(t)$.

There is a spike in $\delta t$ and $\delta \phi$ at around $6 \mathrm{~h}$ from the start of the measurement. During this $100 \mathrm{~s}$ segment, there was a large seismic excitation and our weak photon calibrator signal was overwhelmed by this noise. Therefore, legitimate $\delta t$ and $\delta \phi$ are not available in this short period. When measuring scientific data, the LIGO veto system detects these kind of glitches in the environmental monitors, such as seismometers and issues a veto flag. Therefore, noisy segments like this are not used for data analysis to look for gravitational wave signals.

The mean, error and standard deviation (STD) of $\delta t$ and $\delta \phi$ are listed in table 1 . The errors are estimated by dividing the STD by the square root of the number of segments used for the averaging, because the distributions of $\delta t$ and $\delta \phi$ are well fit with Gaussians. When calculating these numbers, the noisy segments at the beginning and at $6 \mathrm{~h}$ were not used. 


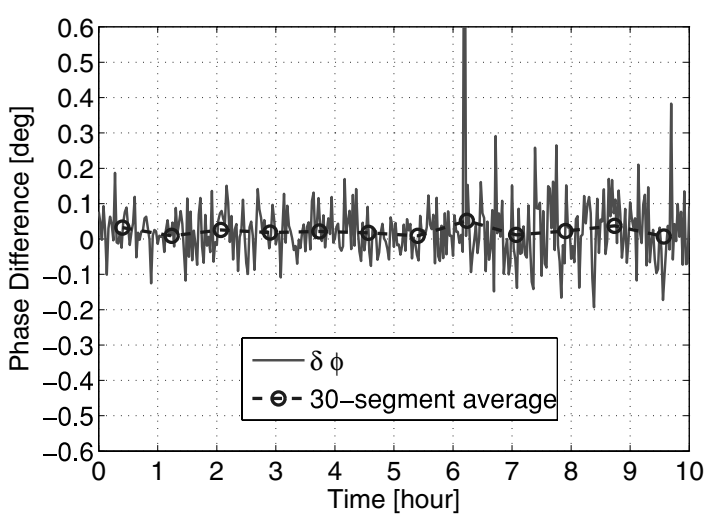

Figure 4. The measured phase difference using the calibrated data.

Table 1. Mean and standard deviation of $\delta t$ and $\delta \phi$ measured between $h(t)$ and $p(t)$.

\begin{tabular}{llllll}
\hline Mean $\delta t$ & Error of $\delta t$ & STD of $\delta t$ & Mean $\delta \phi$ & Error of $\delta \phi$ & STD of $\delta \phi$ \\
\hline $211.4 \mu \mathrm{s}$ & $\pm 0.26 \mu \mathrm{s}$ & $5.0 \mu \mathrm{s}$ & $0.019^{\circ}$ & $\pm 0.0039^{\circ}$ & $0.074^{\circ}$ \\
\hline
\end{tabular}

Table 2. Mean and standard deviation of simulated $\delta t$ and $\delta \phi$.

\begin{tabular}{llll}
\hline Mean $\delta t$ & STD of $\delta t$ & Mean $\delta \phi$ & STD of $\delta \phi$ \\
\hline $212.9 \mu \mathrm{s}$ & $2.5 \mu \mathrm{s}$ & $0.024^{\circ}$ & $0.149^{\circ}$ \\
\hline
\end{tabular}

\section{Simulation}

In figures 3 and 4 the measured quantities fluctuate randomly except for the large glitch. These fluctuations can come either from contamination of the two-tone signal by the random noise of the interferometer or from a real fluctuation of the time delay and the phase difference of the interferometer. The latter can occur when the interferometer's state, such as the alignment of the mirrors, is changed. In order to see the amount of contribution from stationary instrument noise, we generated simulated data which consist of a two-tone signal and white Gaussian noise and measured the time delay and phase difference. The simulated two-tone signal is prepared to have $\delta t=213 \mu \mathrm{s}$ and $\delta \phi=0.019^{\circ}$ (arbitrary choices). The signal and noise amplitudes are chosen to be similar to the real strain data around $110 \mathrm{~Hz}$. The amplitude spectral densities of the real and simulated data are shown in figures 5 and 6 . Since the spectrum of the real data shows bumps around the signal frequencies, we chose the level of the top of the bumps as the level of our white noise. The source of the side lobes around the signal frequencies in figure 5 is most plausibly nonlinearities in the instrument which show up when a strong excitation is applied.

The results of the simulation are shown in figures 7 and 8 . The mean and standard deviations of the recovered $\delta t$ and $\delta \phi$ are shown in table 2. The injected time delay was recovered with good precision. The standard deviations of the time delay and the phase difference are of the same order as the numbers listed in table 1 . Therefore, we believe that the fluctuations observed in figures 3 and 4 are mostly due to the noise of the interferometer. 


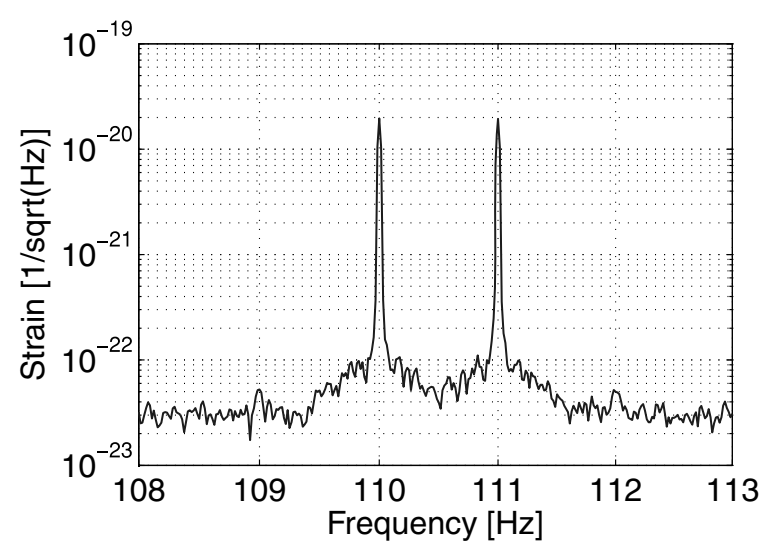

Figure 5. Amplitude spectral density of the strain data around $110 \mathrm{~Hz}$.

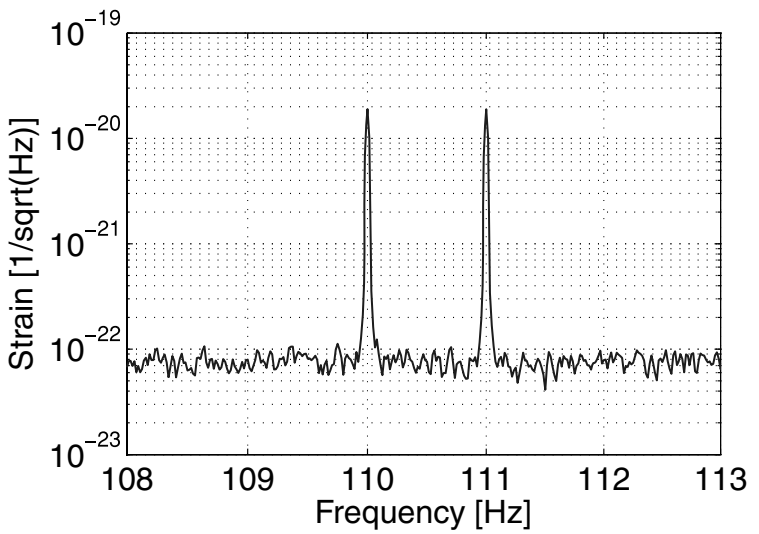

Figure 6. Amplitude spectral density of the simulated data around $110 \mathrm{~Hz}$.

\section{Discussion}

\subsection{Interpretation}

The measured $211.4 \mu$ s time advance in $h(t)$ can be understood as the combination of the delay in the photon calibrator's witness photo detector path and the improper assignment of time delay in the control loop when the preliminary $h(t)$ was generated. The break down of the $211.4 \mu$ s is shown in table 3 .

The timing difference between ADC1 and ADC2 has been monitored by the LIGO timing distribution system. At the time of the measurement, ADC2 was delayed from ADC1 by 25.5 $\mu \mathrm{s}$. This delay combined with a $4.0 \mu$ s delay in the photon calibrator's witness photo detector appears as an apparent advance of $h(t)$ with respect to $p(t)$.

The calibration model used to generate the $h(t)$ was a preliminary one and had errors in the treatment of the time delay. This systematic error is estimated to be a time advance of about $174 \mu \mathrm{s}$. The origin of the $174 \mu \mathrm{s}$ is explained in the following paragraph. In addition, 


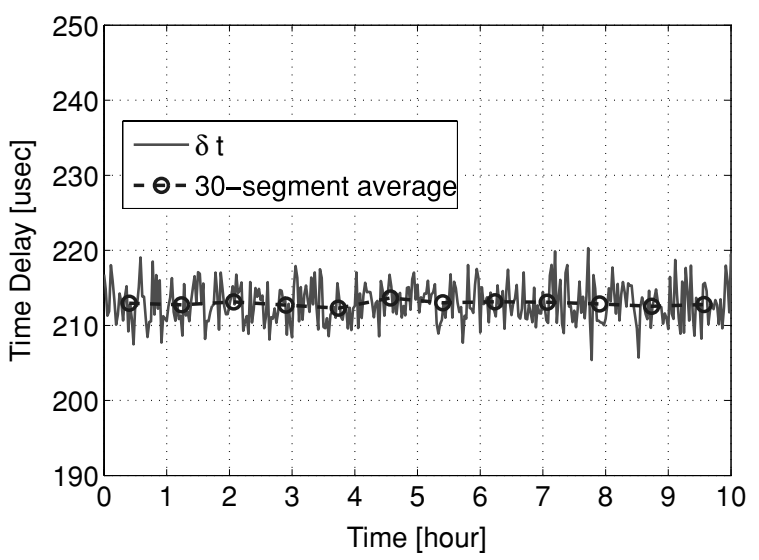

Figure 7. Simulated time delay measurement.

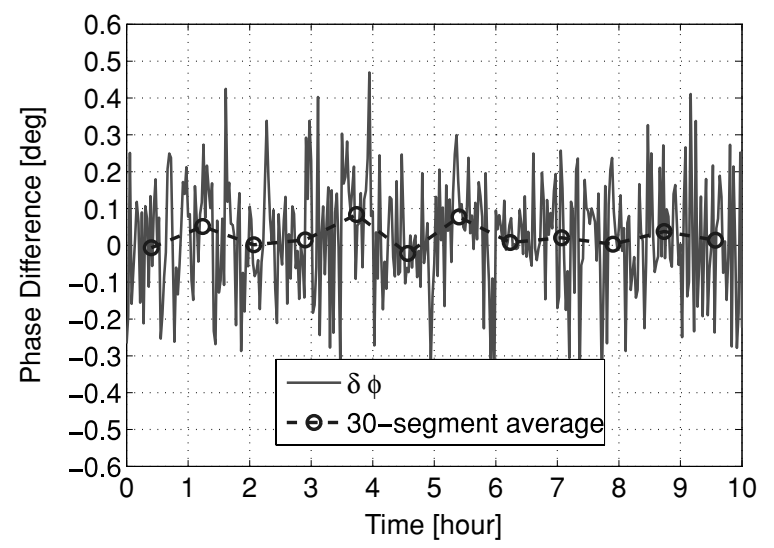

Figure 8. Simulated phase difference measurement.

approximations used for time delays in the preliminary model have an uncertainty of 5-10 $\mu \mathrm{s}$. This is quoted as the uncertainty of the systematic error in table 3.

The expected time delay in the length-control loop of the interferometer consists of a computer processing delay of two samples or $122.1 \mu \mathrm{s}, 30.5 \mu \mathrm{s}$ for the sample and hold delay of the digital-to-analog converters (DACs) used to drive the coils and $13.3 \mu \mathrm{s}$ for the light travel time in the $4 \mathrm{~km}$ arms. Direct measurements [24] of the computer processing and DAC delays are consistent with expectations. In addition, direct measurements of the photodetector response constrain extra delays in sensing the interferometer output to be less than $5 \mu \mathrm{s}$. The expected around-the-loop delay is therefore approximately $166 \mu \mathrm{s}$. The generation of the preliminary $h(t)$ used a $187 \mu$ s delay to match the model of the loop-transfer function with the measured open-loop-transfer function. It includes an extra DAC sample and hold delay of $30.5 \mu \mathrm{s}$, because it was inadvertently included in the model with the wrong sign. This $187 \mu \mathrm{s}$ delay was assigned to the sensing part (the box designated 'interferometer' in figure 2) of the control loop where it had maximal effect on the output. However, as mentioned above, most of the delays in the loop are attributable to the feedback part (e.g. the computer processing 
Table 3. Interpretation of the measured time delay.

\begin{tabular}{lll}
\hline Effect & Value & Uncertainty \\
\hline Measured delay & $+211.4 \mu \mathrm{s}$ & $1 \mu \mathrm{s}$ \\
ADC2 delay & $-25.5 \mu \mathrm{s}$ & $1 \mu \mathrm{s}$ \\
Monitor photodetector delay & $-4.0 \mu \mathrm{s}$ & $2 \mu \mathrm{s}$ \\
Systematic error in the calibration model & $-173.7 \mu \mathrm{s}$ & $10 \mu \mathrm{s}$ \\
Remaining total & $+8.2 \mu \mathrm{s}$ & $10 \mu \mathrm{s}$ \\
\hline
\end{tabular}

and DACs). The correct model, which will be used for the final calibration, assigns this delay, except for the $13.3 \mu$ s of the light travel time, to the coil actuation where it has no effect on the interferometer response. Therefore the preliminary $h(t)$ used in this analysis includes 173.7 (=187-13.3) $\mu$ s of time advance to compensate for the incorrectly assigned time delay in the sensing part.

The remaining discrepancy between the time delay used to match the model and the measured transfer functions $(156.5=187-30.5 \mu \mathrm{s})$ and the sum of the identified delays $(166 \mu \mathrm{s})$ is $9.5 \mu \mathrm{s}$. One possible explanation is the uncertainty in the phase calibration of $h(t)$, which is approximately $50 \mu \mathrm{s}$ at our measurement frequencies, mainly arising from uncertainties in measured parameters. For example, the optical response of the differential arm length shows a pole around $100 \mathrm{~Hz}$ which is due to the optical cavities in the Michelson arms. A $1 \%$ change in the cavity poles would cause a change in the delay of $7 \mu \mathrm{s}$ at $110 \mathrm{~Hz}$.

As shown in table 3, the measured $211.4 \mu$ s time advance is accounted for by the known correction factors with $10 \mu$ s accuracy. This is not significant enough to cause any concern. To better understand the discrepancy, further investigations of the unexplained delay and the influence of the cavity pole will be needed. The original timing requirement is an overall accuracy of $\pm 10 \mu \mathrm{s}$. Our understanding of the time delays in the interferometer and the associated electronics has reached this level.

So far we have not included a correction from ADC1 or the timing-distribution system. These effects are common to both the calibrated $h(t)$ signal and our measurement with the photon calibrator. They should be included when an absolute time stamp is required. The bias from ADC1 is small and will add $3 \mu \mathrm{s} \pm 2 \mu \mathrm{s}$. There is no known bias in the timing-distribution system. Its uncertainty is estimated to be $\pm 2 \mu \mathrm{s}$.

\subsection{Verification of the calibration}

The optical gain of a LIGO interferometer fluctuates during operations due to, for example, alignment fluctuations of the mirrors, or variations of the laser power. Therefore, LIGO's calibration system monitors the interferometer response by injecting calibration signals into the interferometer and by adaptively changing the calibration factors [25]. If the adaptive calibration compensation system develops a problem, it affects $h(t)$ through the alteration of the unity gain frequency in the control loop shown in figure 2. This change in the phase response is detectable by our time delay measurement. Therefore, we can use our method to verify the validity of the adaptive calibration compensation system. To see this, we analysed the same data as used in section 4, but artificially limit the adaptive compensation of the calibration factors by prohibiting more than $20 \%$ of deviation from the normal values.

Figures 9 and 10 show the plots of $\delta t$ and $\delta \phi$ when the restriction was imposed. There is a big jump in $\delta t$ at around $6 \mathrm{~h}$. As discussed before, this was the time when there was a 


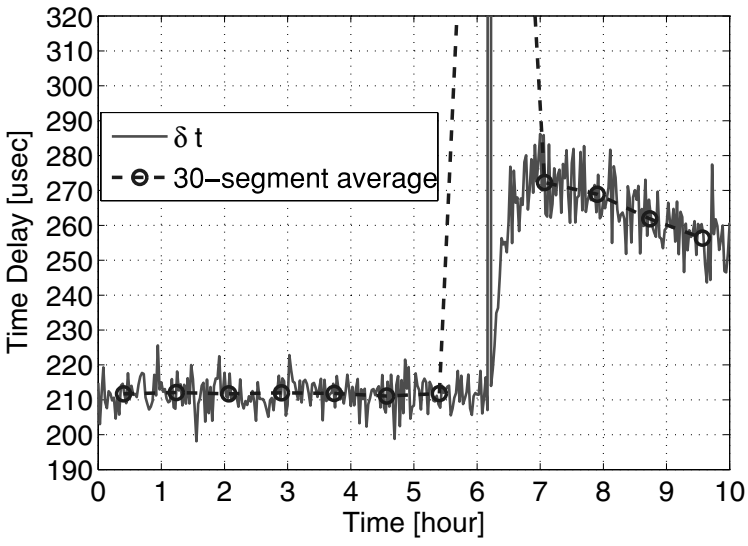

Figure 9. Time delay $\delta t$ between $h(t)$ and $p(t)$ when a restriction is imposed on the adaptive calibration compensation system.

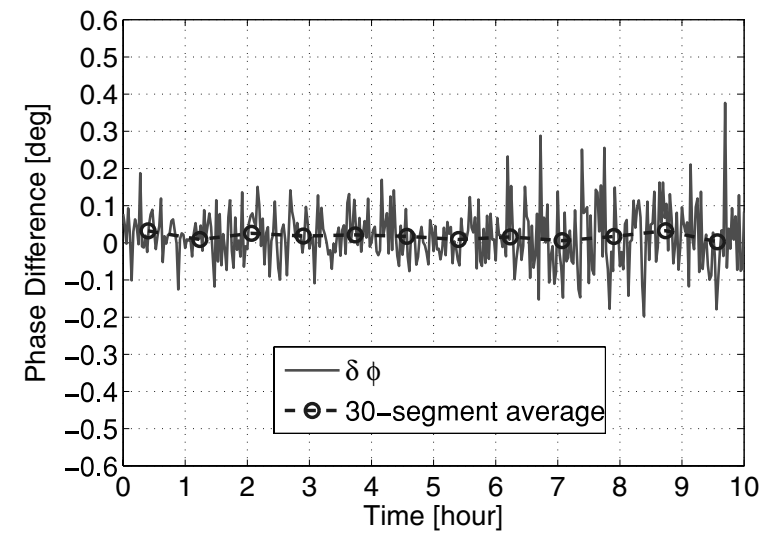

Figure 10. Phase difference $\delta \phi$ between $h(t)$ and $p(t)$ when a restriction is imposed on the adaptive calibration compensation system.

large seismic excitation. The alignment-monitoring system of the mirrors shows a significant shift in the orientation of some of the mirrors. Consequently, this glitch caused a large change in the calibration factor, and the adaptive compensation system could not follow it because of the artificial restriction imposed. This is the cause of the large jump in $\delta t$. As seen in figure 3 , the properly set up adaptive calibration compensation system completely removed the problem under normal conditions.

\subsection{Strength of photon calibrator excitation}

The current LIGO specification on the timing accuracy is $10 \mu \mathrm{s}$. Therefore, it is desirable to be able to monitor the time delay with the resolution of $\sim 1 \mu \mathrm{s}$. As is evident in figure 3 , single $100 \mathrm{~s}$ measurements fluctuate more than $1 \mu \mathrm{s}$, the standard deviation is $5.1 \mu \mathrm{s}$. Hence, we have to average over $\sim 30$ segments to bring the standard deviation below $1 \mu \mathrm{s}$. A 30-segment average takes $3000 \mathrm{~s}$ or $50 \mathrm{~min}$. In order to detect transient events shorter than $3000 \mathrm{~s}$, such as the jump shown in figure 9 , with a resolution of $\leqslant 1 \mu \mathrm{s}$, we would have to increase the signal 
strength of the photon calibrator excitation or have a gravitational wave detector that is ten times more sensitive than initial LIGO was during S5 (e.g., advanced LIGO).

We performed a similar simulation as explained in section 5 with a ten times stronger photon calibrator signal. In this case, the standard deviation of the time delay is reduced to $0.26 \mu \mathrm{s}$. The standard deviation of the phase difference is $0.014^{\circ}$. According to this simulation, we can reduce the error coming from the stationary instrument noise to less than $1 \mu \mathrm{s}$ for $100 \mathrm{~s}$ integration by a signal injection with a ten times larger signal-to-noise ratio. However, we have to be careful when choosing the signal strength because too strong a signal can introduce wide side lobes via the nonlinearity of the detector and contaminate the sensitivity of the interferometer.

\section{Conclusion}

It is crucial for the detection of gravitational waves to accurately calibrate the timing and phase of the calibrated data stream used for gravitational wave searches. We investigated and characterized a method capable of directly measuring the time delay between the force exerted on the test mass of the interferometer and the calibrated data stream, $h(t)$. The measurement performed using the Hanford $4 \mathrm{~km}$ interferometer shows that our method can provide $1 \mu \mathrm{s}$ relative accuracy by averaging over $3000 \mathrm{~s}$ of data. The simulation study shows that we can achieve the same accuracy with a $100 \mathrm{~s}$ long measurement, if we improve the signal-to-noise ratio of the photon calibrator signal by a factor of 10 .

This method is useful not only to determine the time delay of the interferometer, but also to provide a direct and near real-time verification of the calibration system. We have shown agreement between the two methods at the $10 \mu \mathrm{s}$ level. We propose to run this measurement continuously during the operation of the interferometers in future observations.

\section{Acknowledgments}

The authors are grateful for the support of the United States National Science Foundation under cooperative agreement PHY-04-57528 and Columbia University in the City of New York. We are grateful to the LIGO collaboration for their support. We are indebted to many of our colleagues for frequent and fruitful discussion.

The authors gratefully acknowledge the support of the United States National Science Foundation for the construction and operation of the LIGO Laboratory and the Particle Physics and Astronomy Research Council of the United Kingdom, the Max-Planck-Society and the State of Niedersachsen/Germany for support of the construction and operation of the GEO600 detector. The authors also gratefully acknowledge the support of the research by these agencies and by the Australian Research Council, the Natural Sciences and Engineering Research Council of Canada, the Council of Scientific and Industrial Research of India, the Department of Science and Technology of India, the Spanish Ministerio de Educacion y Ciencia, The National Aeronautics and Space Administration, the John Simon Guggenheim Foundation, the Alexander von Humboldt Foundation, the Leverhulme Trust, the David and Lucile Packard Foundation, the Research Corporation, and the Alfred PFF Sloan Foundation.

The LIGO Observatories were constructed by the California Institute of Technology and Massachusetts Institute of Technology with funding from the National Science Foundation under cooperative agreement PHY-9210038. The LIGO Laboratory operates under cooperative agreement PHY-0107417.

This paper has been assigned LIGO Document Number LIGO-P080072-00-Z. 


\section{References}

[1] Hughes S A, Márka S, Bender P L and Hogan C J 2001 New physics and astronomy with the new gravitationalwave observatories Proc. 2001 Snowmass Meeting p 402

[2] Abbott B (the LIGO Scientific Collaboration) 2007 arXiv:0711.3041

[3] Sigg D (the LIGO Science Collaboration) 2006 Class. Quantum Grav. 23 S51-6

[4] Waldman S J (the LIGO Science Collaboration) 2006 Class. Quantum Grav. 233

[5] Abbott B et al (LIGO Scientific Collaboration) 2007 Class. Quantum Grav. 24 5343-69

[6] Abbott B et al 2004 Phys. Rev. D 69102001

[7] Abbott B et al 2005 Phys. Rev. D 72122004

[8] Márka S and Matone L 2006 Searching for cataclysmic cosmic events with a coincident gamma-ray burst and gravitational wave signature Gamma-Ray Bursts in the Swift Era (AIP Conf. Ser. vol 836) ed S S Holt, N Gehrels and J A Nousek pp 605-11

[9] The LIGO Scientific Collaboration 2007 Phys. Rev. D 76062003

[10] Marka S and Mohanty S D 2005 Nucl. Phys. Proc. Suppl. 138 446-8

[11] Mohanty S D et al 2004 Class. Quantum Grav. 21 S765-74

[12] Arnaud N, Barsuglia M, Bizouard M-A, Brisson V, Cavalier F, Davier M, Hello P, Kreckelbergh S and Porter E K 2004 Astropart. Phys. 21 201-21

[13] Abbott B et al 2008 Astrophys. J. 681 1419-30

[14] Landry M 2008 Searches for continuous gravitational waves with LIGO and GEO600 40 Years of Pulsars: Millisecond Pulsars, Magnetars and More (AIP Conf. Ser. vol 983) (New York: AIP) pp 627-9

[15] Abbott B et al 2008 Astrophys. J. 683 L45-9

[16] Abbott B et al 2007 Astrophys. J. 659 918-30

[17] Goetz E et al 2009 in preparation

[18] Sigg D 1997 LIGO Document T970101

[19] Rakhmanov M, Romano J D and Whelan J T 2008 Class. Quantum Grav. 25

[20] Fritschel P, Bork R, Gonzalez G, Mavalvala N, Ouimette D, Rong H S, Sigg D and Zucker M 2001 Appl. Opt. 40 4988-98

[21] Hamming R W 1997 Digital Filters 3rd edn (New York: Dover)

[22] IEEE standard 10571994 IEEE Standard for Digitizing Waveform Recorders

[23] Handel P 2000 IEEE Trans. Instrum. Meas. 49 1189-93

[24] Smith N and Sigg D 2008 LIGO Document T080039-00-D

[25] Dietz A, Garofoli J, González G, Landry M, O’Reilly B and Sung M 2006 LIGO Document LIGO-T050262-01 